EPJ Web of Conferences 92,02098 (2015)

DOI: $10.1051 /$ epjconf/ 20159202098

(C) Owned by the authors, published by EDP Sciences, 2015

\title{
Electrodiffusion Method of Near-Wall Flow Diagnostics in Microfluidic Systems
}

\author{
J. Tihon a, V. Pěnkavová, P. Stanovský, and J. Vejražka \\ Institute of Chemical Process Fundamentals, ASCR, Rozvojová 135, 16502 Prague, Czech Republic
}

\begin{abstract}
The electrodiffusion technique has been mostly used for the near-wall flow diagnostics on large scales. A novel technique for fabrication of plastic microfluidic systems with integrated metal microelectrodes (called technique of sacrificed substrate) enables us to produce microfluidic devices with precisely shaped sensors for wall shear stress measurements. Several micrometer thick gold sensors, which are built-in a plastic substrate, exhibit good mechanical resistance and smoothness. Proper functioning of prepared chips with microsensors has been first tested in various calibration experiments (polarization curve, sensor response to polarization set-up, steady flow calibration, temperature dependence of diffusivity). Our first results obtained for separating/reattaching flow behind a backward-facing step and for gas-liquid Taylor flow in microchannels then demonstrate its applicability for the detection of near-wall flow reversal, the delimitation of flow recirculation zones, and the determination of wall shear stress response to moving bubbles. Other applications of these sensors in microfluidics (e.g. characterization of liquid films, capillary waves, bubbles or drops) can be also envisaged.
\end{abstract}

\section{Introduction}

The electrodiffusion technique [1,2], which has been until now used only for the near-wall flow diagnostics on larger scales, can be promising for both, scientific experiments and microdevice diagnostics. The electrodiffusion sensors prepared by the photolithography have been already applied to investigate the structure of near-wall turbulence in channel flows [3] and their applications in microfluidic systems have been just considered [4-6]. New microfabrication techniques enable us to produce microfluidic devices with precisely shaped microelectrodes. It offers a possibility to use such electrodiffusion sensors for near-wall flow investigations in various microfluidic systems (e.g. in complex channel geometries often used as micromixers, or possibly in multiphase microfluidic systems dealing with bubbles and drops.)

\section{Electrodiffusion technique}

The electrodiffusion sensor works as a small electrode, on which a fast electrochemical reaction takes place, when a small polarization voltage is applied to it. The electric current passing through the electrode is the measured quantity. The current signal $I$ provided by a simple strip probe is controlled by convective diffusion and depending on the wall shear rate $\gamma$ according to the well-known Léveque formula

$$
I=0.807 n F c_{0} w l^{2 / 3} D^{2 / 3} \gamma^{1 / 3},
$$

where $n$ is the number of electrons involved in the electrochemical reaction, $F$ is the Faraday constant, $l$ is the length of the strip sensor in the mean flow direction, $w$ is its width, $c_{0}$ is the bulk concentration of the active ions, and $D$ is their diffusivity in the solution. The main advantage of this technique is that the wall probes provide information about the flow in the near wall region without any disturbances imposed on the studied velocity field.

The Léveque formula (Eq.1) relates the instantaneous values of wall shear rate and limiting diffusion current only if measured flow fluctuations are slow enough (quasi-steady). For proper use of sensors under unsteady flow conditions, it is necessary to consider their dynamic response. The approximate model [7] suggested for description of the probe dynamic behavior is represented by a simple formula

$$
\gamma=k_{s}^{-3}\left(I^{3}+2 k_{t}^{2} \frac{d I}{d t}\right)
$$

This semi-empirical differential equation contains two constants, easily accessible from the steady flow calibrations.

\footnotetext{
${ }^{\mathrm{a}}$ Corresponding author: tihon@icpf.cas.cz
} 
If the exact shape of a probe and the diffusivity and concentration of ions involved in the electrode reaction are known, they can be also calculated from the theoretical relationships. Considering a single strip probe, the first constant $k_{s}$ is obtained from the steady state solution (Eq.1), which provides

$$
k_{s}=0.807 z F c_{0} w l^{2 / 3} D^{2 / 3}
$$

and the second one $k_{t}$ can be determined from the known solution of the unsteady diffusion at the beginning of the transient process after the probe polarization switch-on

$$
I=k_{t} t^{-1 / 2}
$$

with

$$
k_{t}=z F c_{0} w l \sqrt{D / \pi}
$$

If flow reversal occurs in the near-wall flow region and additional information on the flow direction is needed, a two-segment probe [8] is usually applied. The instantaneous flow direction is determined by simple comparison of current signals obtained from the upstream $\left(I_{1}\right)$ and downstream $\left(I_{2}\right)$ probe segment. The rear segment (in respect to the actual flow direction) is located in a concentration shade of the front one and thus is giving the lower signal. Therefore, a possible near-wall flow reversal is detected from a sudden change of $I_{1} / I_{2}(\mathrm{t})$ current ratio signal (from $I_{l}(\mathrm{t})>I_{2}(\mathrm{t})$ to $I_{2}(\mathrm{t})>I_{l}(\mathrm{t})$ ). This criterion can be easily used to localize the stagnation points between steady recirculation-flow regions.

Classical versatile sensors are fabricated from platinum foils or wires, which are glued into a stainless steel tube acting as an auxiliary electrode. A novel technique for fabrication of plastic microfluidic systems with integrated metal microelectrodes (called technique of sacrificed substrate) has been developed at ICT Prague [9]. Several micrometer thick gold sensors built-in into a plastic substrate exhibit good mechanical resistance and smoothness.

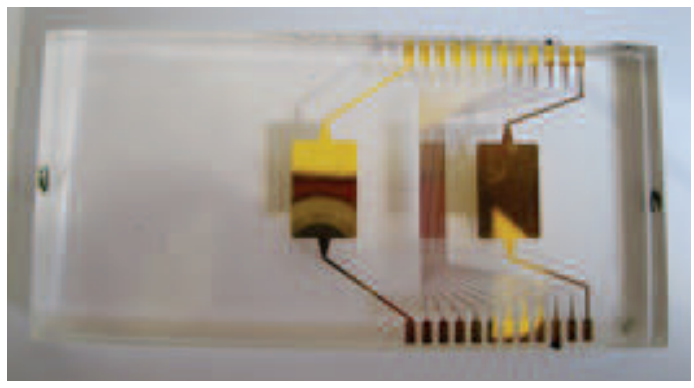

Figure 1. Measuring chip with the gold strip sensors ( 20 cathodes located between 2 large anodes).

\section{Experiments in microchannels}

\subsection{Sensors and electrolyte}

A measuring chip with sensors fabricated by the method of a sacrificed substrate consists of an array of gold strips embedded into a Plexiglas plate (see Figure 1). Twenty sensing cathodes (160 $\mu \mathrm{m}$ strips) separated by $40 \mu \mathrm{m}$ insulating gaps are located between two large anodes (10 $\mathrm{mm}$ strips). The design of the chip enables us to use cathodes as individual (strip) or double (two-strip) sensors. The plate with microelectrodes serves as a top cover (roof wall) of several microchannel devices.

Water with equimolar $0.002 \mathrm{M}$ potassium ferrocyanide and ferricyanide is used as a suitable electrochemical system and $0.2 \mathrm{M}$ potassium sulfate as a supporting electrolyte. The resulting solution has a density of $\rho=1024 \mathrm{~kg} / \mathrm{m}^{3}$ and a kinematic viscosity of $v$ $=1.06 \mathrm{~mm}^{2} / \mathrm{s}$ at a reference temperature of $22{ }^{\circ} \mathrm{C}$. The polarization voltage of $-0.6 \mathrm{~V}$ applied between sensing electrodes and counter-electrodes assures that ferricyanide ions reduce on the electrode surface and the resulting current is limited by the convective diffusion. An in-house electrodiffusion device is used to apply the polarization voltage, to convert currents through the individual sensors into voltage signals, and to amplify them. The device is controlled by a computer, which also records the measured signals.

\subsection{Experimental apparatus}

Two microchannels with rectangular cross-sections (200 and $800 \mu \mathrm{m}$ in height and $10 \mathrm{~mm}$ in width) are applied for the calibration of sensors. Their length of $80 \mathrm{~mm}$ is sufficient to achieve developed flow conditions at the measuring locations. The active surface of sensors is delimited by a sealing located between the microchannel and measuring chip and thus forming side walls of microchannels. Both these parts are fixed into a clamp table, which enables to shift the chip with sensors in respect to the channel inlet (see Figure 2). Two gear pumps Heidolph and Micropump are used to supply the electrolyte into the microchannel, covering the range $Q_{L}$ from 7 to $500 \mathrm{ml} / \mathrm{min}$.

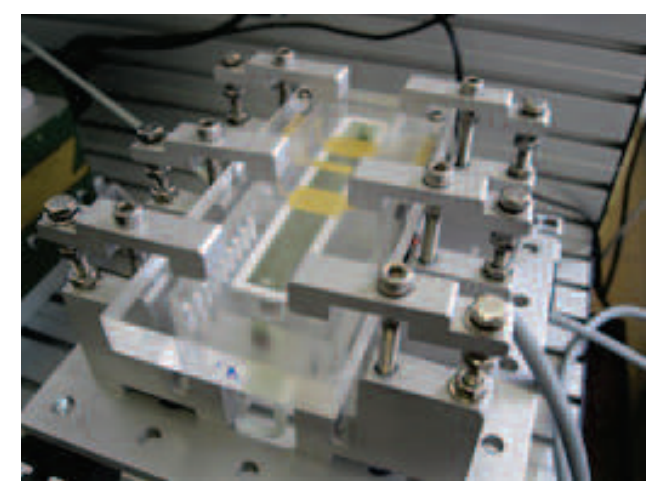

Figure 2. Experimental apparatus.

A stainless steel foil (thickness of $400 \mu \mathrm{m}$ ) is used to form a backward-facing step on the roof wall of the larger microchannel. Two other microchannels with a rib and a step on the bottom walls are used to study separating and reattaching flows. A capillary $(20 \mu \mathrm{m}$ i.d. $)$ is placed into inlet tubing to inject air into the microchannel and thus produce gas-liquid Taylor flow. The bubble growth is controlled by adjusting the pressure and opening times of two (inlet and vent) electromagnetic valves. 


\section{Results and discussion}

\subsection{Sensor testing and calibration}

Proper functioning of prepared sensors has been first tested by the measurement of polarization curves (see Figure 3). As currents measured at moderate polarization voltages (around $\mathrm{U} \sim-0.5 \mathrm{~V}$ ) are limited by diffusion of the active ions towards the sensor surface, the observed polarization curves are characterized by distinct plateaus. The current at plateaus is sensitive to actual flow conditions (i.e. wall shear rate magnitudes) in the microchannel. The polarization voltage of $-0.6 \mathrm{~V}$ has been chosen for calibrations and measurements.

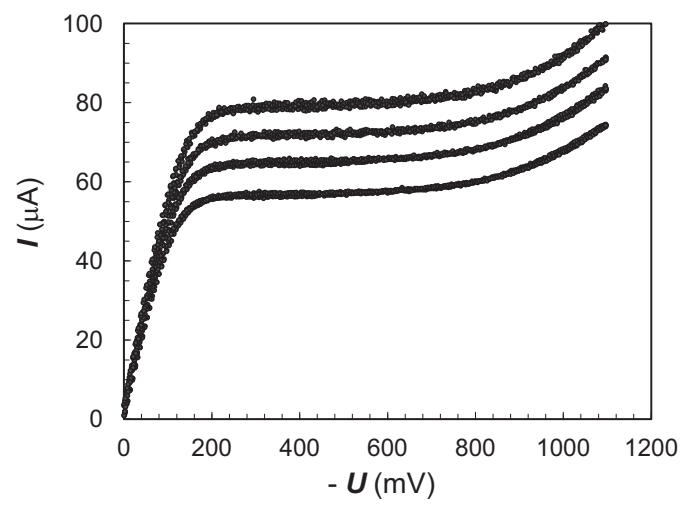

Figure 3. Limiting diffusion currents measured at different flow rates (for $\gamma=3120,4690,6250$, and $7810 \mathrm{~s}^{-1}$ ).

Practically same polarization curves have been obtained for all tested sensors. The individual currents from sensors for the same flow rates have been differentiated less than $0.5 \%$. The results of sensor calibration carried out under steady laminar flow conditions are shown in Figure 4. The measured currents follow very well a cubic root dependence on the wall shear rate prescribed by Eq.1. The calibration constant $k_{s}=3.52 \mu \mathrm{A} . \mathrm{s}^{1 / 3}$ is determined from data fit (dash line). As the electrolyte concentration and sensor dimensions are known, Eq. 3 provides for the diffusion coefficient of ferricyanide ions an estimate of $D=6.710^{-10} \mathrm{~m}^{2} / \mathrm{s}$. This value (measured at temperature of $T=22{ }^{\circ} \mathrm{C}$ ) is in a good agreement with the previous experimental findings [10].

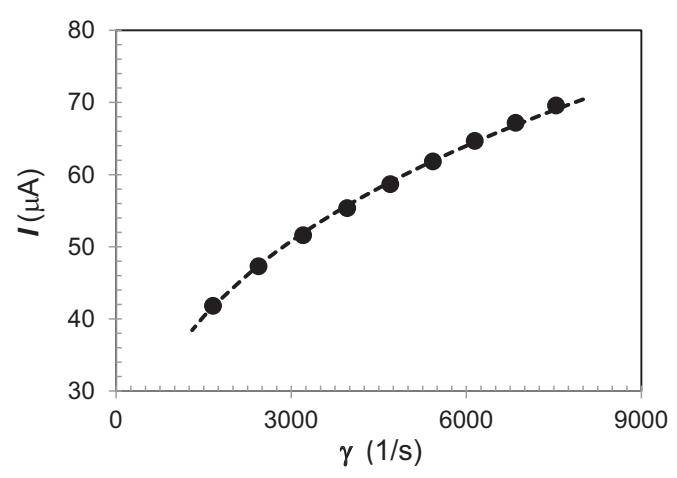

Figure 4. Sensor calibration at the steady flow conditions (dash line stands for data fit: $I=3.52 \gamma^{1 / 3}$ ).
The transient current response to a set-up of polarization voltage, obtained for the sensor in stagnant electrolyte, is presented in Figure 5. The measured data are controlled by unsteady diffusion at the beginning of transient process and can be fitted well by the Cottrell asymptote (Eq.4). The calibration constant $k_{t}=4.7 \mu \mathrm{A} . \mathrm{s}^{1 / 2}$ obtained from this dynamic calibration then provides the diffusion coefficient of $D=7.310^{-10} \mathrm{~m}^{2} / \mathrm{s}$ (Eq.5). As this value agrees satisfactorily with that obtained from the steady calibration, the proper functioning of sensors is verified.

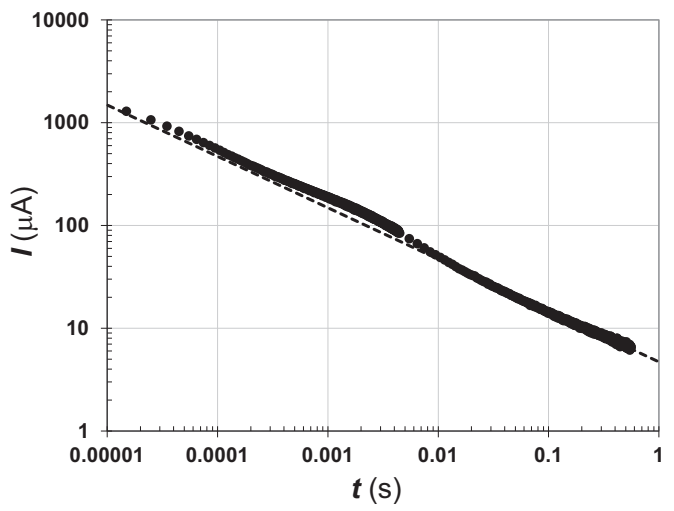

Figure 5. Transient current response to the setting-up of polarization voltage measured in stagnant electrolyte (dash line stands for low current data fit: $I=4.7 t^{-1 / 2}$ ).

\subsection{Backward-facing step flow}

To measure the wall shear rate profile behind a backward-facing step (channel height $H=800 \mu \mathrm{m}$, step height $h=400 \mu \mathrm{m}$ ), sensors are used as double (two-strip) sensors and the measuring chip is subsequently fixed at four positions (the first sensor at $x_{0}=0,4,8,12 \mathrm{~mm}$ ) to cover the measuring range $x / h$ from 0 to 40 . At low flow rates (for $\mathrm{Re}=2 U H / v<600$ ), the backward-facing step flow is found to be steady without any significant fluctuations. The position of reattachment is fixed and the wall shear rate should be equal to zero there.
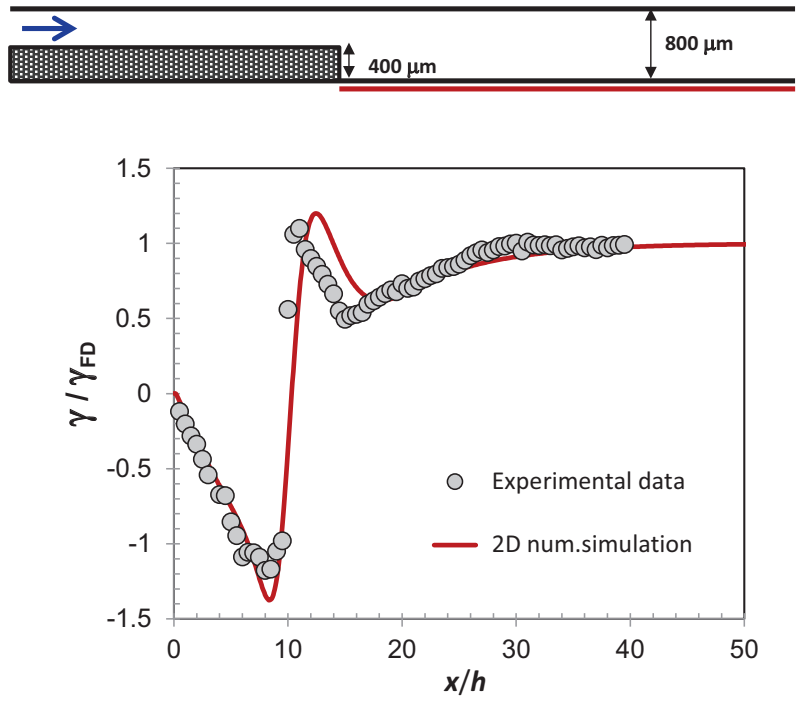

Figure 6. Wall shear rate profile behind the step at $\mathrm{Re}=625$ $\left(\gamma_{0}=2930 \mathrm{~s}^{-1}\right.$ for the fully developed channel flow) 
The typical wall shear rate profiles measured under still laminar flow conditions (at $R e=625$ ) are presented in Figure 6. As the flow in a flat channel is predominantly two-dimensional, the obtained experimental data are in good agreement with the results of our previous 2D numerical simulations [11]. The axial profile obtained at the bottom wall exhibits a strong negative peak corresponding to flow reversal inside a primary recirculation zone (for $x / h$ from 0 to 9.75 ), which is followed by a region of slow recovery to the channel flow (for $x / h$ from 9.75 to 40 ). The exact position of reattachment point is determined from measured $I_{2} / I_{1}(x / h)$ profile $\left(I_{2} / I_{1}=1\right.$ for $\left.x=x_{r}\right)$. The wall shear rate profile measured at the roof wall shows that the near-wall flow is very close to separation there.

As soon as the flow separation at the roof leads to the inception of a recirculation eddy, the flow configuration with two flow-recirculation regions becomes unsteady and flow fluctuations originated from the separated shear layer are progressively amplified in the recirculation regions. Unsteady character of near-wall flow inside the reattachment region is shown in Figure 7.

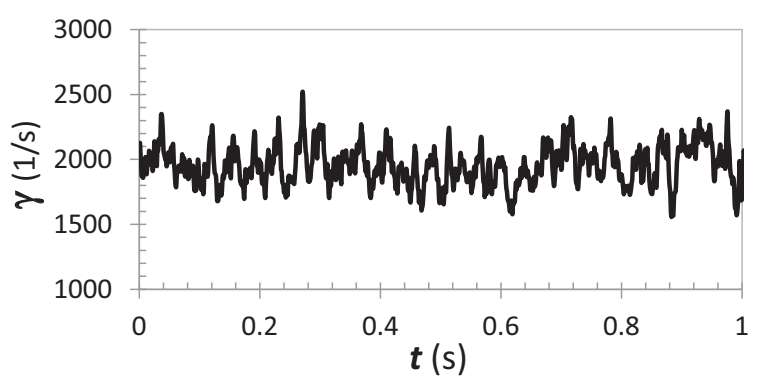

Figure 7. Time trace of wall shear rate measured behind the reattachment at $x / h=13.5$ and $R e=1105$.

As the wall shear rate magnitude $(\gamma \sim U / H)$ in microchannels is much higher than in common channels, also the frequency range of observed flow fluctuations is significantly higher. Therefore, to find frequency scaling of fluctuations from the spectral analysis, the dynamic behavior of sensors has to be considered. To capture correctly possible small and high-frequency fluctuations induced by a micro backward-facing step, smaller sensor sizes are also needed. This experiment is now in preparation.

\subsection{Flow over a transverse rib}

In micro heat transfer applications a typical modification of the channel wall is an orthogonal barrier, effectively creating a step in the fluid flow path that significantly enhances heat transfer by modifying the flow field [12]. To validate the results of numerical simulations on the effect of a flow-disturbing rib on heat transfer rates in the microscale, the electrodiffusion measurements of wall shear rate profiles in the microchannel above a rectangular rib have been carried out. The experiments have been done for Reynolds numbers ranging up to 1500 and the typical results are presented in Figure 8. Wall shear rate at the roof wall exhibits a peak value just in front of the rib and then a rapid drop behind the rib. Both, the experimental and numerical results are in a quite good agreement. Only the appearance of flow separation at the roof wall is predicted little bit earlier in simulations. In the experiments the roof eddy has been observed for $\operatorname{Re}>850$. The detailed results of this flow investigation, especially the effect of key design parameters on the separating/reattaching flow over the rib can be found in the recent paper [13].

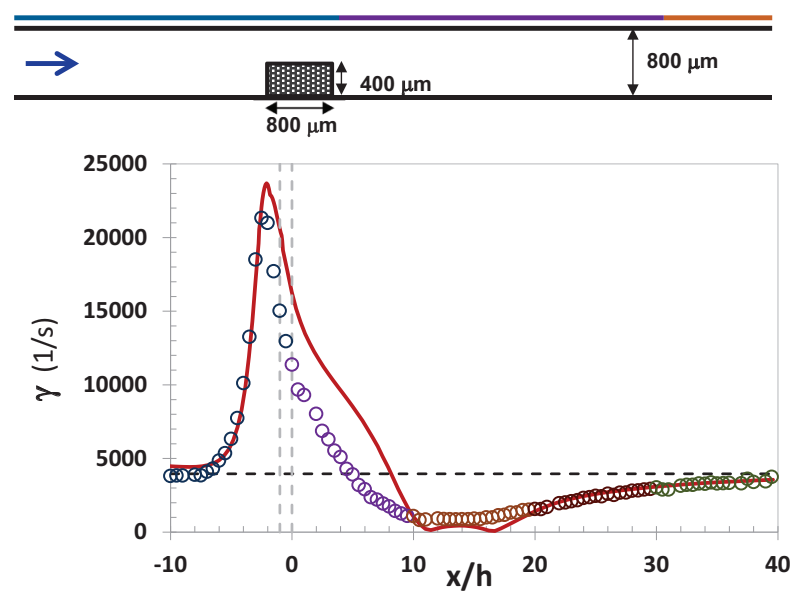

Figure 8. Wall shear rate profile on the roof wall above the rib measured at $\mathrm{Re}=840$. Experimental data (symbols) are compared with the numerical results (red line).

\subsection{Gas-liquid Taylor flow}

Gas-liquid Taylor flow experiments have been carried out in the microchannel with $H=800 \mu \mathrm{m}$, which has been oriented either horizontally or vertically. Typical electrodiffusion data measured during the rise of a single large bubble in the vertical micro-channel are shown in Figure 9. In this case, a flat bubble $\left(V_{B}=60 \mu \mathrm{l}\right)$ with a typical "bullet-like" outline was rising in co-flowing liquid $\left(U_{L}=0.0156 \mathrm{~m} / \mathrm{s}\right)$. The current signals recorded from two sensors $\left(1^{\text {st }}\right.$ and $20^{\text {th }}$ sensor from the array) are presented in Figure 9a and the time series of wall shear rate corresponding to the first sensor is shown in Figure 9b.
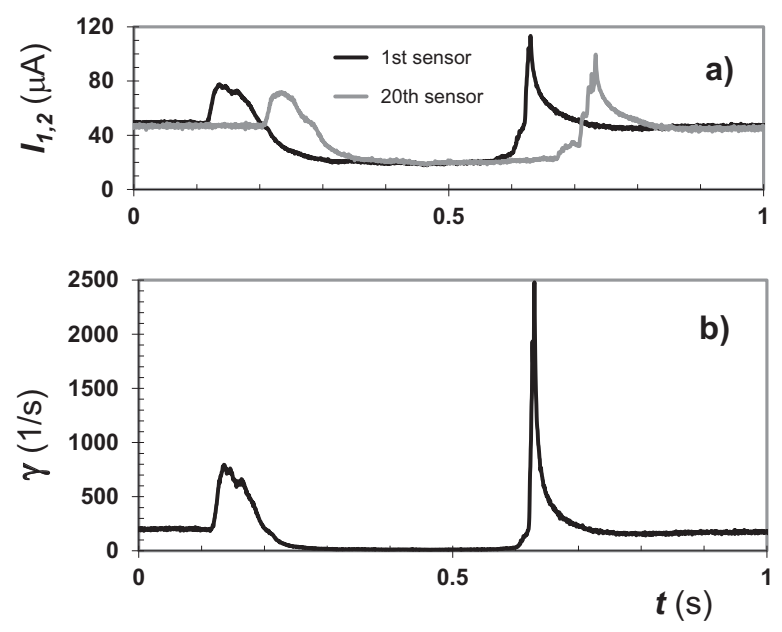

Figure 9. Response of measured signals to a rising bubble $\left(H=0.8 \mathrm{~mm}, W=10 \mathrm{~mm}, U_{B}=0.042 \mathrm{~m} / \mathrm{s} V_{B}=60 \mu \mathrm{l}\right.$ ): (a) currents from two sensors, (b) wall shear rate calculated from the primary current signal. 
The bubble rise velocity $\left(U_{B}=0.0418 \mathrm{~m} / \mathrm{s}\right)$ is calculated from the time shift $\Delta t=91 \mathrm{~ms}$ between two current signals measured by the sensors separated by the distance $\Delta x=3.8 \mathrm{~mm}$. Even though the channel height is less than $1 \mathrm{~mm}$, buoyancy still play an important role in the vertical arrangement (bubble velocity in stagnant liquid is $U_{B 0}=0.03 \mathrm{~m} / \mathrm{s}$ ). The trace of wall shear rate corresponding to the near-wall flow around the rising bubble exhibits two peaks (the first at bubble front, the second at bubble back), which are separated by the region of small wall shear rates (almost stagnant liquid film located between the flat side of bubble and the wide wall of micro-channel).

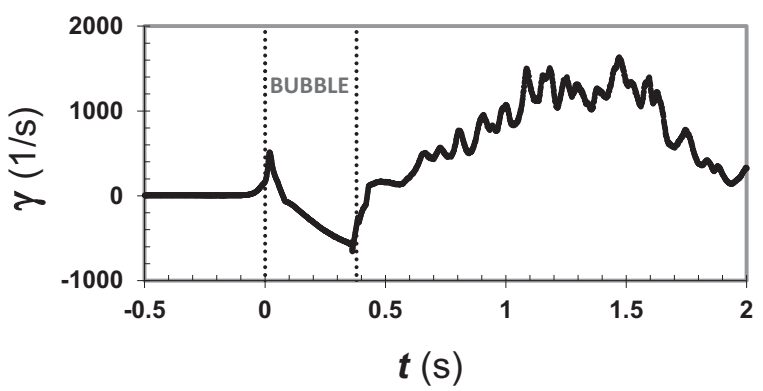

Figure 10. Wall shear rate induced by a rising bubble in stagnant water in the vertical rectangular channel ( $\left.H=4 \mathrm{~mm}, W=50 \mathrm{~mm}, V_{B}=8 \mathrm{ml}, U_{B}=0.156 \mathrm{~m} / \mathrm{s}\right)$.

This wall shear rate profile differentiates significantly from those measured in large channels, where the strong reverse flow in the liquid film under the bubble and highly unsteady flow behind the bubble are observed. It is documented in Figure 10, where the wall shear rate data [14] measured in a rectangular channel with the crosssection 4 × $50 \mathrm{~mm}$ are shown.

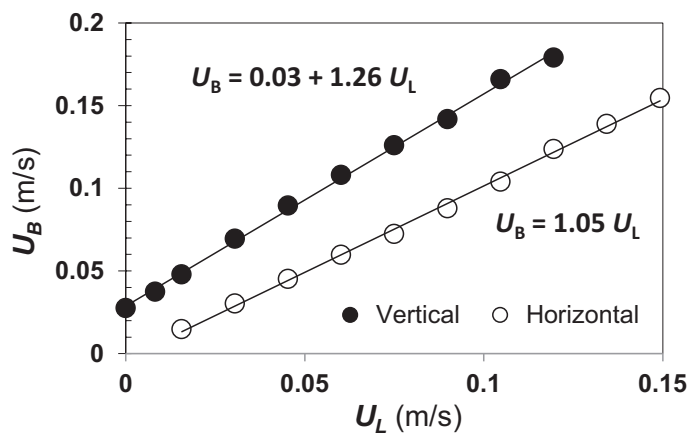

Figure 11. Velocities of large bubbles translating in co-flowing liquid measured for the vertical and horizontal orientation of the microchannel.

The velocity of bubble translation $U_{B}$ in liquid coflowing with the mean velocity $U_{L}$ can be expressed as a sum of two components: the bubble velocity in stagnant liquid $U_{B o}$ and a component proportional to the mean liquid velocity

$$
U_{B}=U_{B o}+C_{0} U_{L} \text {. }
$$

As seen in Figure 11, this linear relationship holds also in microchannels. However, the distribution coefficient $C_{0}$ is significantly smaller than in large channels, where its value is controlled by the maximum liquid velocity at the channel center-line (thus for the laminar flow in a flat channel $\left.C_{0} \sim 1.5\right)$. Magnitude of $C_{0}$ is found to be sensitive also to the channel inclination. In the horizontal microchannel the bubble velocity is only $5 \%$ higher than the mean velocity of co-flowing liquid.

\section{Conclusions}

The chip with electrodiffusion sensors fabricated by the method of a sacrificed substrate proved to be a reliable platform for the flow diagnostics in microfluidic devices. Our results obtained in rather complex microchannel flow configurations (a backward-facing step, a transverse rib, and gas-liquid Taylor flow) demonstrate its applicability for the detection of near-wall flow reversal, the delimitation of flow-recirculation zones, and the determination of wall shear stress response to moving bubbles. Other applications of these sensors in microfluidics (e.g. characterization of falling liquid films, waves, bubbles or drops) are also envisaged.

\section{Acknowledgement}

The support by Czech Science Foundation GACR through the contract $\mathrm{P} 101 / 12 / 0585$ is gratefully acknowledged.

\section{References}

1. J.R. Selman, Ch.V. Tobias, 1978. Mass-transfer measurements by the limiting-current technique, in Advances in Chem. Engineering (Acad.Press, NY, 1978)

2. T.J. Hanratty, J.A. Campbell, Fluid mechanics measurements (Washington, Hemisphere, 1983)

3. C. Deslouis, B. Tribollet B, J. Tihon, J. NonNewtonian Fluid Mech. 123, 141 (2004)

4. X. Illa, O. Ordeig, D. Snakenborg, A. RomanoRodriguez, R.G. Compton, J.P. Kutter, Lab on a Chip, 10, 1254 (2010)

5. N.A. Mouheb, A. Montillet, C. Solliec, J. Havlica, P. Legentilhomme, J. Comiti, J. Tihon, Microfluid Nanofluid, 10,1185 (2011)

6. K. Abi-Samra, T.H. Kim, D.K. Park, N. Kim, J. Kim, H. Kim, Y.K. Cho, M. Madou, Lab Chip, 13, 3253 (2013)

7. J. Tihon, J. Legrand, H. Aouabed, P. Legentilhomme, Exp. Fluids, 20, 131 (1995)

8. J.S. Son, T.J. Hanratty, J Fluid Mech, 35, 353 (1969)

9. W. Schrott, M. Svoboda, Z. Slouka, D. Snita, Microelectron Eng, 86, 1340 (2009)

10. E. Eroglu, S. Yapici, O.N. Sara, J Chem Eng Data, 56, 3312 (2011)

11. J. Tihon, V. Penkavova, J. Havlica, M. Simcik, Exp Therm Fluid Sci, 40, 112 (2012)

12. I.A. Stogiannis, A.D. Passos, A.A. Mouza, S.V. Paras, Appl. Therm. Eng., 59, 717 (2013)

13. I.A. Stogiannis, A.D. Passos, A.A. Mouza, S.V. Paras, V. Penkavova, J. Tihon, Chem. Eng. Sci. 119, 65 (2014)

14. J. Tihon, V. Penkavova, J. Vejrazka, Int.J.Multiphase Fow, 67, 76 (2014) 